\title{
Correlation Between Substrate Morphology and the Initial Stages of Epitaxial Organic Growth: PTCDA/Ag(111)
}

\author{
Pierre L. Lévesque, ${ }^{\dagger \dagger}$ Helder Marchetto, ${ }^{\dagger, \S}$ Thomas Schmidt, ${ }^{*},, \|$ Florian C. Maier, ${ }^{\|, \perp}$ \\ Hans-Joachim Freund, ${ }^{\dagger}$ and Eberhard Umbach ${ }^{\dagger, \|}$ \\ ${ }^{\dagger}$ Chemische Physik, Fritz-Haber-Institut der Max-Planck-Gesellschaft, Faradayweg 4-6, 14195 Berlin, Germany \\ ${ }^{\ddagger}$ Département de Chimie, Université de Montréal, Montréal, Québec H3C 3J7, Canada \\ ${ }^{\S}$ ELMITEC Elektronenmikroskopie GmbH, Albrecht-von-Groddeck-Straße 3, 38678 Clausthal-Zellerfeld, Germany \\ "Experimentelle Physik, Universität Würzburg, Am Hubland, 97074 Würzburg, Germany \\ ${ }^{\perp}$ IEK-5 Photovoltaik, Forschungszentrum Jülich GmbH, Wilhelm-Johnen-Straße, 52425 Jülich, Germany
}

Supporting Information

ABSTRACT: We have investigated the initial growth of the first two epitaxial layers of PTCDA on a $\mathrm{Ag}(111)$ surface consisting of a distribution of flat (111) terraces, separated by single atomic steps or step bunches with a few point defects. By utilizing the low-energy electron microscopy (LEEM) technique in both bright and dark field modes, we are able not only to follow the growth of the first layers but also to distinguish between different rotational and mirror domains and their influence on the growth of subsequent layers. Thus, we learn much about diffusion lengths and barriers, domain sizes, and about the influence of domain boundaries and nucleation centers. The results give deep insight into the growth dynamics, the influence of step orientation, and

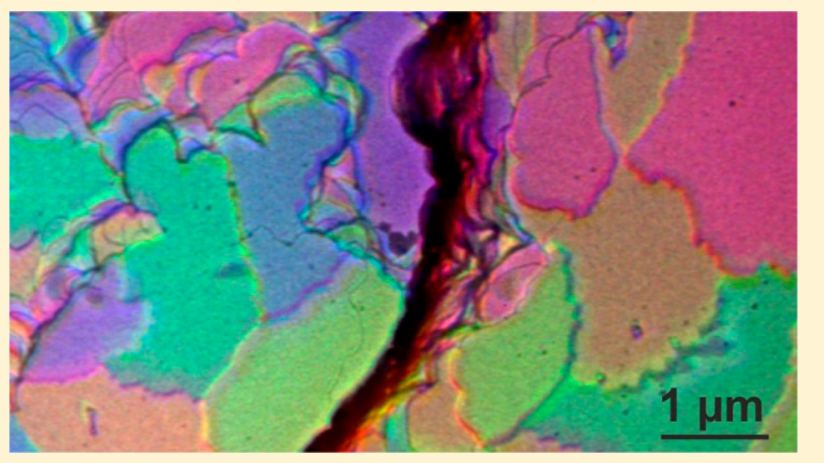
the quality of the resulting epitaxial layers and lead to the conclusion that the morphology of the substrate surface is likely to be the most influential parameter for the homogeneity of epitaxial layers.

\section{INTRODUCTION}

The understanding and tailoring of thin organic films and their interfaces to inorganic materials is a field of high scientific interest. The reason is not only that the field comprises an enormous amount of different, yet unexplored systems and many poorly or not understood phenomena. The reason is also that organic devices such as organic photovoltaics, ${ }^{1,2}$ organic field effect transistors, ${ }^{3}$ and organic light-emitting displays ${ }^{4}$ strongly depend on the electric and optical properties of such films that in turn depend strongly on the structure and morphology of the films as well as on the interfaces between films and substrates or contacts. Often it is not really understood why properties such as photo- or electroluminescence yield or electric conductivity drastically change as a function of preparation conditions or why unexpected failures occur. As example with respect to the system studied here, we just refer to earlier work where it has been found that the photoluminescence yield of a film of 30 ordered layers of 3,4,9,10-Perylenetetracarboxylicacid-Dianhydride (PTCDA) on $\operatorname{Ag}(111)$ decreases by a factor of about 20 when the preparation temperature of the layer is changed by $80 \mathrm{~K}$ (from 230 to 310 $\mathrm{K})$ although most experimental techniques are unable to detect a difference of layer morphology, structure, or electronic properties. $^{5}$
Therefore, it is worthwhile studying the growth properties of organic films, the influence of the substrate and the influence of the preparation parameters. Of course, these strongly depend on the molecular structure and the interaction between the molecules and between molecules and substrate. This may lead to completely different behavior even if only the size of the molecule, the orientation of the substrate or a functional group has been changed. Hence, it appears impossible to develop general rules and recipes because each system requires its own treatment. However, from detailed studies of selected systems one can gain deep insight in a few systems from which sufficient experience may be derived that is useful for tackling other systems in a focused way.

The model system selected for the present investigation has been chosen as one extreme case of organic/inorganic layer systems, a system that in principle is capable to develop "true" epitaxial growth. We here define epitaxial growth as growth of a highly ordered thin organic film of several layers thickness (not just a monolayer ${ }^{6}$ ) that is in registry with an ordered substrate, that is, there is a commensurate relationship between the

\footnotetext{
Received: July 6, 2016

Revised: August 2, 2016

Published: August 3, 2016
} 
geometric structure of the substrate and that of the organic film. Because the surface unit cells of inorganic crystals and those of organic crystals are usually very different, and because the preferential bonding of most organic molecules to most substrates is either so weak that the molecules do not care about the substrate surface structure or so strong that they do not care about neighboring molecules, the number of possible epitaxial systems is rather limited.

The model system chosen here, PTCDA on $\operatorname{Ag}(111)$, is a potential candidate for epitaxial growth because the unit cells of the $\operatorname{Ag}(111)$ surface and the (102) plane of the two modifications of PTCDA crystals are nearly commensurate, that is, the lattice mismatch is in the $2-3 \%$ range. ${ }^{7,8}$ Moreover, the (102) plane of PTCDA crystals has two molecules per unit cell that lie exactly in this plane, thus providing the ideal orientation because adsorbed PTCDA molecules on $\operatorname{Ag}(111)$ also lie flat on the substrate., ${ }^{9,10}$ This is well-known and has been investigated in great detail in many studies by many authors, and even the (epitaxial) growth properties have been studied with different methods and by different groups. ${ }^{7,11-13}$ The focus of the present paper is the extension of the previous knowledge concerning two important questions: (a) how strongly do surface steps or step bunches on the substrate influence the growth of epitaxial organic layers and (b) how large can crystallites (ordered homogeneous domains) grow in view of the fact that already the first organic layer can form 198 different domains $(3 \times 2 \times 33=198$ domains, rotational, mirror, and translational domains, ${ }^{7,8}$ and each further layer adds a factor of 4 (including alpha and beta modification)?

The ideal and perhaps only method that can answer these two questions simultaneously is low-energy-electron-microscopy (LEEM). This method yields structural data, can distinguish between different rotational domains, can discern first, second, and higher layers, has a sufficiently high lateral resolution to see single atomic steps on the surface, and can be used in a dynamic mode. The latter property means that one can watch the organic film growing, can vary parameters like temperature and deposition rate, and can monitor the growth of several domains simultaneously under identical conditions thus providing a kind of statistical information. The present analysis hence presents a number of new results that considerably add to the existing knowledge. ${ }^{7,11-13}$

\section{EXPERIMENTAL SECTION}

The instrument used for the LEEM experiments is the SMART spectro-microscope built within a collaboration of several German groups from the universities Würzburg, Erlangen, Clausthal, the Fritz-Haber Institute of the Max-Planck Society, the Zeiss company, and BESSY (now HZB). ${ }^{14-16}$ The microscope is installed at the BESSY-II storage ring of the Helmholtz-Center Berlin for Material and Energy (HZB).

SMART is equipped with an aberration corrector, compensating simultaneously both, chromatic and spherical aberrations, ${ }^{19-19}$ and with an imaging energy filter. A lateral resolution of better than $2.6 \mathrm{~nm}$ could be experimentally demonstrated in LEEM. ${ }^{20,21}$ The design of the specimen chamber enables the deposition of, for example, organic material under grazing incidence $\left(20^{\circ}\right)$ on the sample surface at the measurement position in front of the objective lens. Therefore, the growth of the PTCDA film could be directly observed in real time. For the deposition a Knudsen cell type evaporator was used. The deposition rate was set to about 0.08 $\mathrm{ML} / \mathrm{min}$ ( $\mathrm{ML}=$ monolayer coverage $)$. One monolayer corresponds to the deposited amount required to saturate the first PTCDA layer (of parallel oriented molecules) on the surface at $330 \mathrm{~K}$ (at this temperature desorption can be neglected). The base pressure of the measurement chamber was $<3 \times 10^{-10}$ mbar. A potential influence of the instrumental setup on the growth has been carefully checked and could be excluded. ${ }^{11}$ The kinetic energy of the electrons at the sample surface was reduced below $5 \mathrm{eV}$, which is about the threshold below which no beam damage is observed.

$\mathrm{A} \operatorname{Ag}(111)$ single crystal oriented within an accuracy better than $0.2^{\circ}$ has been cleaned by cycles of Ar-sputtering $(600 \mathrm{eV}$, $1 \mu \mathrm{A}, 5 \times 10^{-5} \mathrm{mbar}, 15 \mathrm{~min}$, room temperature) with subsequent annealing at $700-800 \mathrm{~K}$ for about $15 \mathrm{~min}$. The cleaning progress was checked by photoelectron emission microscopy, LEEM, low-energy electron diffraction (LEED), and X-ray photoelectron spectroscopy.

\section{RESULTS AND DISCUSSION}

3.1. Overview and Contrast Mechanism. In Figure 1a, we start with a LEEM image from a bare surface area (field of
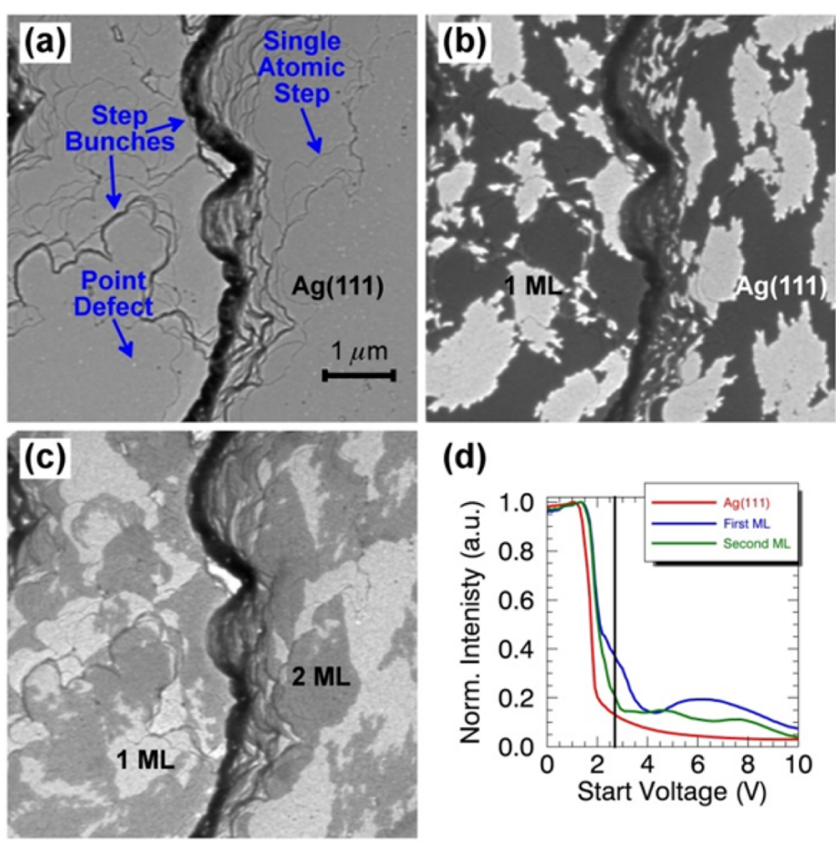

(d)

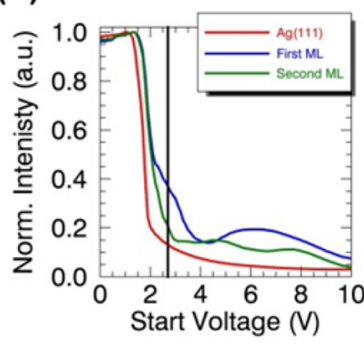

Figure 1. (a) LEEM image of clean $\operatorname{Ag}(111)$. Single atomic steps appear as thin dark lines and step bunches as thick dark lines. Point defects are visible as bright or dark dots. (b) PTCDA (0.5 ML) on $\mathrm{Ag}(111)$. The first layer appears brighter than the $\mathrm{Ag}(111)$ substrate. (c) PTCDA (1.5 ML) on $\mathrm{Ag}(111)$. The second layer is darker than the first layer and brighter that the $\mathrm{Ag}(111)$ substrate (not visible in panel c). (d) Intensity of $\mathrm{Ag}(111)$, first (1 ML), and second (2 ML) layer PTCDA as a function of start voltage. All images of the present paper have been taken at an energy of $2.7 \mathrm{eV}$ (black line) if not otherwise stated.

view: $5 \times 5 \mu \mathrm{m}^{2}$ ) selected such that all substrate surface structures that could be important for the layer growth mechanism are observable: smooth (111) terraces (right side, lower left side), point defects, single atomic steps, bunches with few steps, and a bunch with a large number of steps in the middle (from top to bottom). In Figure 1b, half a monolayer has been deposited on the bare surface, and in Figure 1c half of the second layer has been deposited on the saturated monolayer. In Figure 1b, the PTCDA monolayer islands 
appear bright on the dark substrate and in Figure 1c the second layer appears darker than the first layer. Steps and in particular step bunches are still visible but seem to be less pronounced. At this point, we like to stress that LEEM is able to see even monatomic steps and to distinguish between different kinds of step bunches, which is important for the study of their influence on the growth kinetics. Moreover, LEEM can distinguish the first few PTCDA layers, which in our case grow layer-by-layer (see, for example, refs 7 and 11).

The contrast mechanism of LEEM is based on the backscattering and interference of slow electrons that are reflected from a surface and detected. The mechanism has been described several times. ${ }^{22-28}$ Of course, backscattering intensity and interference effects strongly depend on the primary energy of the electrons that in turn determines the detected signal as can be seen in Figure 1d. There the normalized intensity of the integrated signal is plotted as a function of primary voltage for the three cases shown in Figure $1 \mathrm{a}-\mathrm{d}$. For the clean $\operatorname{Ag}(111)$ surface, the backscattered intensity drastically drops above a threshold of (nominal) $1.3 \mathrm{~V}$ because electrons penetrate into the Ag sample and get lost. (The absolute value of the voltage has no direct meaning because it is the difference between surface potential and the potential of the field emitter tip.)

For the two other cases, the backscattering intensity changes: a shift of the drop voltage by about $0.3 \mathrm{~V}$ toward higher voltage indicates that the work function of the selected sample area has increased by about that value due to chemisorption of the PTCDA monolayer on $\operatorname{Ag}(111)$ (the integral work function change might be slightly different because the selected area may not be representative for the whole sample due to the large step bunch). A slight backshift by $30 \mathrm{mV}$ induced by the second layer indicates that the second layer is only weakly bound to the first.

In addition to the energy shift of the intensity drop, there are intensity oscillations at higher voltages with maxima at about 3 and $6.5 \mathrm{~V}$ for the first and at about 2.5, 4.7, and 7.5 V for the second layer. These are clear examples of interference effects stemming from the interference of the backscattered electrons from the various layers. These intensity oscillations can be utilized to optimize the contrast difference between the three cases as shown in Figure 1d. In the present case (Figures $1 \mathrm{a}-\mathrm{c}$ and 2-7), we selected a primary voltage of $2.7 \mathrm{~V}$ (black vertical line) at which the clearest difference between the three cases is seen (and no beam damage is observable). Indeed, the first layer (blue curve) has the highest intensity at that voltage, followed by the second layer (green curve) and the bare surface (red curve). For a primary voltage of $6-7 \mathrm{~V}$ the contrast situation would have been similar, whereas, for instance, at 4.5 $\mathrm{V}$ the first and second layers would not have been discernible.

The option that a LEEM instrument can also be used to investigate the local geometric structure by low energy electron diffraction (small spot LEED) and that LEEM in the dark-field mode can be utilized to distinguish different rotational/mirror domains and observe their growth separately has been introduced earlier. ${ }^{11}$ We will make use of this unique property of LEEM instruments below but mention here that in the present study we did not observe geometric structures other than those found on $\operatorname{Ag}(111)$ using conventional integral LEED, spot profile analyzing LEED (SPALEED), or STM. ${ }^{6-8,29,30}$

3.2. Growth of the First Layer. Next we study the growth of the first layer in more detail. Generally, the PTCDA molecules evaporated onto the Ag sample at about room
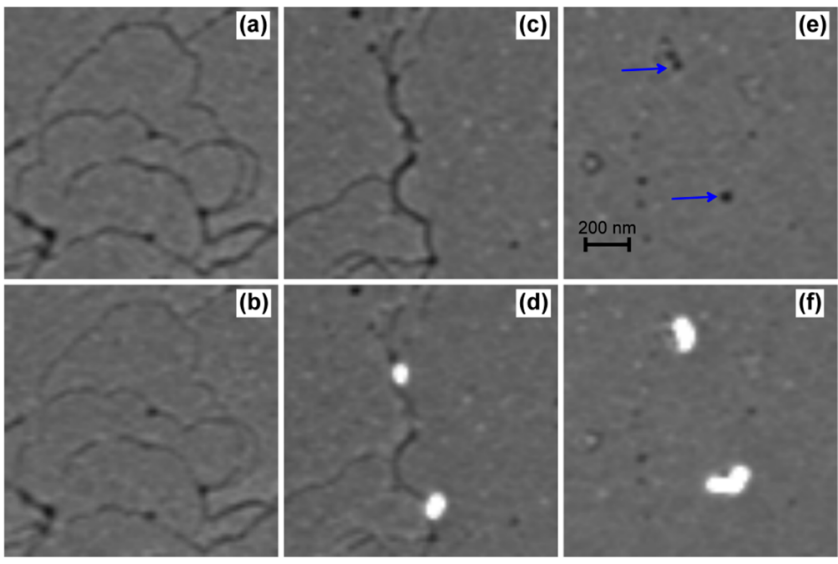

Figure 2. LEEM images to highlight the initial stages of PTCDA adsorption on $\mathrm{Ag}(111)$. In the first row the coverage is zero, in the second row the coverage is less than $0.005 \mathrm{ML}$. The comparison of (a) and (b) shows that single atomic steps and step bunches appear thinner and lighter after decoration by PTCDA. Images (c) and (d) show that nucleation of the PTCDA (bright white islands) occurs at step edges, and images (e) and (f) show that nucleation can also occur at point defects (highlighted by two blue arrows). Nucleation at steps usually occurs on the lower side of the step. Nucleation at point defects is mostly observed on larger terraces.

temperature diffuse over long distances (several micrometers) before they take a fixed adsorption site. ${ }^{11,29}$ In the present case, we observed (see movies in the Supporting Information), that immediately after opening the shutter of the evaporation source nearly nothing, especially no nucleation of islands, occurred apart from an intensity change at the steps and step bunches (compare Figure 2a,b). These become lighter indicating that their local surface potential changes, which is apparently due to preferential adsorption of the first molecules on these steps and step bunches. This finding is consistent with the previous observation by $\mathrm{STM}^{8,31}$ that PTCDA deposition on stepped $\mathrm{Ag}(111)$ surfaces leads to preferential adsorption of PTCDA on two or more monatomic Ag steps and to bunching of adjacent $\mathrm{Ag}$ steps. We assume that before the delayed onset of (observable) nucleation not only steps and step bunches are decorated but also a "reservoir" of highly mobile PTCDA precursor molecules is filled. These diffuse rapidly on the sample surface as kind of a lattice gas that is commonly found for small adsorbates in the study of adsorption kinetics. ${ }^{32-36}$

After a delayed onset (see Figure 2c-f), nucleation preferentially starts at steps, step bunches (compare Figure $2 \mathrm{c}, \mathrm{d}$ ) or point defects (compare Figure $2 \mathrm{e}, \mathrm{f}$ ). The initial nuclei are often a few micrometers apart from each other (see, e.g., Figure $1 \mathrm{~b}$ ). On small terraces (a terrace is defined here as smooth $\operatorname{Ag}(111)$ area surrounded by steps or step bunches; "small" means less than $1 \mu \mathrm{m}$ wide), only one nucleus is found while on large terraces ("large" means several micrometers) more than one nucleus usually occurs. We observed that on small terraces the nucleation often starts on the lower side of a step bunch while on large terraces nucleation may also start at point defects or even at points where no defect is discernible.

With further deposition of PTCDA molecules the initial nuclei (islands) continue growing. It is interesting to note that nearly no new nuclei are added but that preferentially the already existing islands grow in size. The growth usually develops on the terrace where the nucleation started, that is, within the apparent limits set by step bunches. In the first layer 
regime, a given island may grow either along or perpendicular to the step bunch where nucleation started; these directions seem to be stochastically chosen, and the growth direction may even change during growth. The edges of the islands sometimes look fractal but before the monolayer is completed all coves and holes become filled.

Numerous interesting details can be observed when watching the movie (see Supporting Information). Of course not all observable details are representative for the entire surface, but some of those, which appear to happen more often, will be mentioned. Step bunches appear to be strong barriers, whereas (precursor) molecules easily diffuse across them and islands after nucleation usually do not extend over these barriers. The step bunches become already covered in the initial stage of deposition $^{31}$ (causing the delayed onset of nucleation and the change of electron reflectivity) but the filling of spaces between step bunches and islands preferably occurs at the end of monolayer adsorption. Monoatomic steps also appear to hinder the island growth but not as strictly as step bunches because sometimes monatomic steps are overgrown by an expanding island (see Figure 3). In this case, a domain from a large terrace

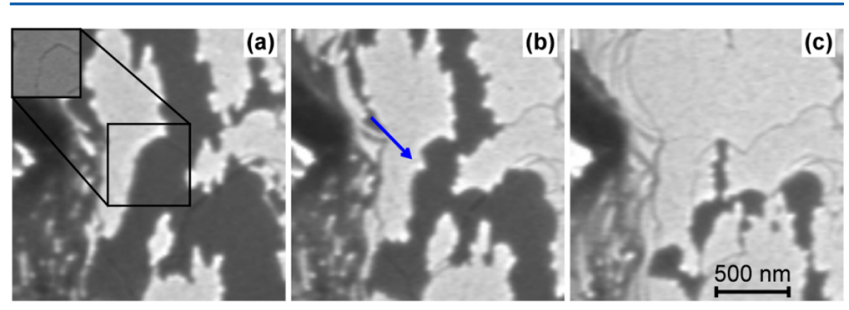

Figure 3. LEEM images of the intermediate stages of PTCDA growth on $\mathrm{Ag}(111)$. The sequence $(\mathrm{a}-\mathrm{c})$ shows the growth of a single domain over a substrate atomic step (images a and b) which is clearly visible in the inset in (a) showing the same area of the clean surface. The PTCDA domain on the upper side of the step grows over the step to the lower terrace indicated by the blue arrow in (b) thereby conserving the rotational orientation. When this domain encounters another domain growing from another nucleation center with different rotational orientation, a rotational domain phase boundary is formed. Image (c) displays the almost-formed boundary, running vertically, just before closure. The coverages for this sequence are $0.56,0.70$, and $0.88 \mathrm{ML}$

overgrows the step and continues growing on the adjacent terrace (see blue arrow) until it reaches a second step or forms a domain boundary with a neighbored island that grows on the same terrace.

The next question to be answered is that of the nature of these islands and domains. From LEED and STM studies ${ }^{8,37}$ it is well established that the monolayer of PTCDA on $\operatorname{Ag}(111)$ can have 198 different domains, 6 of which are discernible by diffraction methods: 3 rotational times 2 mirror domains (the 33 translational domains are not discernible by diffraction methods but could play an important role for the surface structure). Because each rotational/mirror domain has its own (discernible) superstructure fingerprint, the diffraction pattern of the surface usually has a rich pattern of diffraction spots since integral methods sum over all domain patterns.

In the present LEEM apparatus, we have the chance to distinguish the six domains by using the so-called dark-field method. ${ }^{22,38}$ In this case, a small aperture is introduced into one of the diffraction planes within the LEEM instrument such that certain areas of the diffraction pattern are selected (ideally one spot), that is, the corresponding electrons can pass the aperture while all others are blocked. Thus, in the final image plane (real space) those areas that belong to the selected superstructure spot (corresponding to one of the rotational/ mirror domains) appear bright while the others are suppressed. In reality, the finite aperture also allows some electrons from other domains to pass the aperture such that the nonselected domains are still visible but with significantly reduced intensity. When images from different positions of the aperture are compared one can clearly distinguish the six domains and can easily label each observed domain by its rotational/mirror orientation. In our case, we have labeled them with different colors (see Figures 4, 6, and 7).

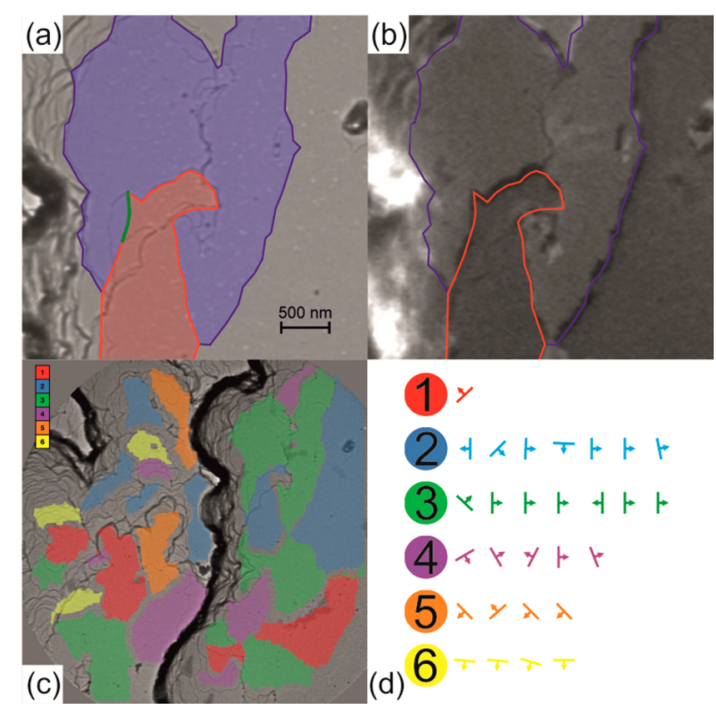

Figure 4. (a) Bright-field LEEM image of the clean $\mathrm{Ag}(111)$ surface (left) and (b) dark-field LEEM image of the same surface region covered by one layer of PTCDA. In the two images, two rotational domains are highlighted in red and blue. The green line in (a) shows the region where the rotational domain boundary formed. Image (c) displays the $\mathrm{Ag}(111)$ surface covered by a monolayer of PTCDA; the largest rotational domains are identified by dark-field images and are identified by different colors. (d) The step orientations where nucleation of the different domains started are indicated.

With the information on the symmetry character of each domain, we can gain more insight. First of all, we found that all domains are equally probable, at least if we evaluate large areas and many domains. Second, we observed that on small and medium terraces usually only one symmetry type of domain develops that can expand over adjacent terraces, while on large terraces different rotational/mirror domains may exist (in different colors in Figure 4) resulting from different nucleation sites. Third, domain boundaries mostly exist between rotational/mirror domains. This means that on small and mediumsized terraces only one domain develops which is limited by step bunches of the substrate and which upon completion of the monolayer covers the entire terrace. On large terraces, the different nuclei growing together have only $17 \%$ probability to be of the same type. Only in the latter case (apart from the situation at steps or bunches) one can observe that different translational domains may also form a domain boundary because the chance that the molecules of both adsorbate domains are exactly in registry is only $3 \%$.

Because some domains nucleate at steps or step bunches the question arises whether the step direction or the step structure 
does have an influence on the selection of the domain symmetry. We have compared the symmetry of the domains that nucleated at steps or step bunches with the orientation of the steps at the nucleation site. The result of this evaluation is shown in Figure 4d; it clearly indicates that in most cases a strong correlation exists between step direction and domain orientation. At present, we cannot conclude on a strict correlation between step direction and domain orientation, because during our experiments we did not pay attention to this aspect and hence did not adjust the resolution accordingly. Moreover, because on the present surface, steps and step bunches are generally curved, errors can be made by selecting the appropriate tangent corresponding to the step orientation. Nevertheless, from the observed strong correlation, we estimate that an enantiomeric selection can be achieved, for example, by a preselection of the step orientation. The latter can be engineered by cutting the sample such that the orientation of the surface normal is a few degrees off the [111] direction that after cleaning and annealing leads to a regular pattern of parallel and equally spaced monatomic steps as shown for $\operatorname{Ag}(111) .{ }^{31} \mathrm{It}$ would be an interesting experiment to see whether by proper selection of the step direction and terrace length, only one type of domain, that is, one enantiomer, would grow on the surface.

The observation of step bunch decoration as primary step, followed by nucleation at step bunches and the observation of preferential selection of rotational domains by the step direction is fully compatible with STM experiments for PTCDA on stepped $\mathrm{Ag}(111)$ surfaces $^{31}$ which showed that the molecules first adsorb on facets (i.e., step bunches in the present case) due to energetic reasons and that the bonding to the steps actually leads to step bunching and to a selection of specific facets with specific orientation and with one type of PTCDA domain. It should be noted, however, that the STM experiments were performed at room temperature after annealing of the layer thus representing a state close to the thermodynamic minimum while the present experiments were recorded during deposition and hence may be strongly influenced by kinetic processes.

Another interesting observation can be made shortly before completion of the monolayer. Above a coverage of about 0.9 ML at which some holes in the first layer still need to be filled, nucleation of the second layer already sets in and second layer domains start to grow. For high deposition rates, the two layers compete for the incoming molecules and grow simultaneously. This process is expected to continue for higher layers such that a relatively rough multilayer far from the ideal layer-by-layer growth is formed. However, the formation of the second layer is metastable as long as holes in the first layer exist because the bonding to the substrate is significantly stronger than that to the first layer. In Figure 5, we demonstrate that after closing the shutter at a dose equivalent to about a monolayer the second layer islands disappeared at the expense of the monolayer holes that became filled. The occurrence of such a kind of Ostwald ripening process leads to the conclusion that better layer-bylayer growth can probably be achieved (at least in the few layer regime) by using small deposition rates, perhaps interrupted by short annealing, that is, ripening cycles.

3.3. Growth of the Second Layer. The second layer grows on top of the first layer, and its growth is strongly influenced by the domain boundaries of the first layer (which occur mainly at step bunches of the substrate). Actually there are nearly no cases observed where domain boundaries of the first layer are overgrown by islands of the second layer. Thus,

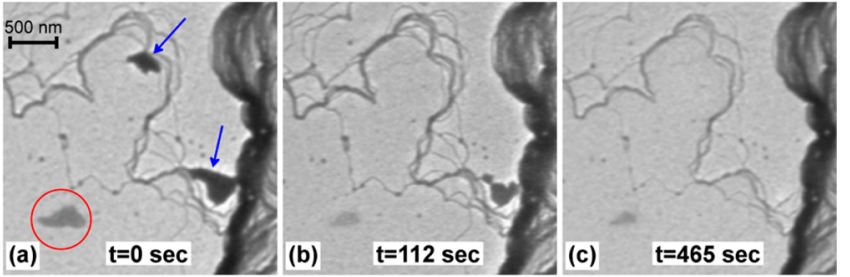

Figure 5. Evolution of the $\mathrm{Ag}(111)$ surface covered by 1.01 ML of PTCDA after deposition (closure of the shutter at $t=0 \mathrm{~s}$ ). In (a), a second layer domain (red circle) coexists with two regions of uncovered $\mathrm{Ag}$ (arrows). One of these visible holes is closed within about $2 \mathrm{~min}$ (b). Finally, after less than $8 \mathrm{~min}$ (c), the first layer is completed and the second layer islands have nearly disappeared.

most domains of the first layer are covered by equally large domains of the second layer. From detailed STM experiments, we know that the molecules of the second layer are in registry with those of the first layer. ${ }^{8}$ Actually, two types of PTCDA crystal structures exist, $\alpha$ and $\beta$ modification, ${ }^{39,40}$ which are distinguished by different relative positions of the molecules in adjacent layers (i.e., different stacking) and by slightly different lattice parameters. The lattice parameters of the PTCDA monolayer on $\operatorname{Ag}(111)$ are closer to those of the (102) plane of the $\beta$-modification that leads to the observation ${ }^{7}$ and assumption here that the positions of the molecules of the second layer are equivalent to those of the $\beta$-modification. If this is the case we should find a similar distribution of rotational domains in the second layer as in the first layer which is indeed the case (see Figure 6).

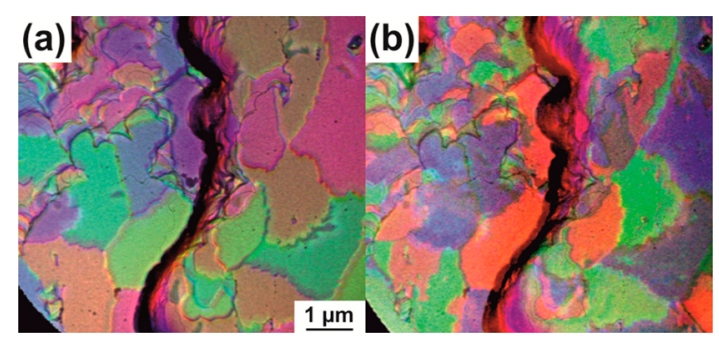

Figure 6. Rotational domains of the first layer (a) and second layer (b) of PTCDA on $\operatorname{Ag}(111)$. The dark-field images were taken at $E_{\text {kin }}=2.7$ and $1.4 \mathrm{eV}$, respectively. Different rotational domains were identified as described in the text and are indicated by different colors. Note that the colors of the two layers are independent, that is, equal colors do not mean equal orientation.

In Figure 6, we compare the rotational domains of the first layer (Figure 6a) with those of the second layer (Figure 6b) from the same area on the sample. It is clearly seen that most first layer domains are covered by equally large second layer domains at the same position corroborating the above statement. However, it can happen that on large first layer domains two or more different rotational domains of the second layer are being formed. This can be seen for the large island in the lower left corner (brown in first layer) or for the green island in the middle near the bottom. At first glance, this appears surprising because one might expect that the first layer determines the growth of the second. However, in fact the molecules in the second layer may have four different positions with respect to the first layer, two belonging to the $\alpha$ - and two to the $\beta$-modification. Even if the $\beta$-modification is preferred due to energetic reasons (the lattice misfit induced by the 
substrate surface is about $2 \%$ for the $\beta$ - and about $3-4 \%$ for the $\alpha$-modification ${ }^{7}$ ), two alternative positions for the second layer remain. Hence at least two different rotational domains of the second layer with respect to the first layer appear possible and are observed in a few cases. Thus, we must conclude that the number of domains slightly increases for the second layer. At present, we have no information whether this behavior continues for higher layers or whether, for example, a possibly increased diffusion length and reduced barrier height of domain boundaries might lead to larger and hence less domains for higher layers.

Next we try to find out which influence domain boundaries between equal rotational domains have, for example, boundaries between translational domains. In Figure 7, two

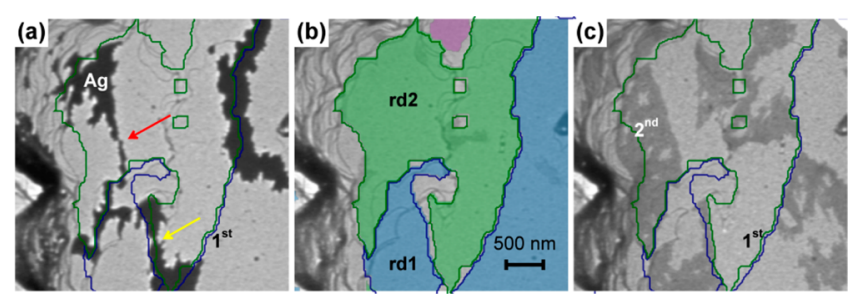

Figure 7. Growth of the second layer of PTCDA on the rotational domains of the first layer. (a) The first layer of PTCDA on $\operatorname{Ag}(111)$ just before completion. Two different domain boundaries are indicated by arrows: one between two rotational domains with the same rotational orientation (red arrow) and one between two different rotational domains (yellow arrow). (b) The two different rotational domains ( $\mathrm{rd} 1$ and $\mathrm{rd} 2$ ) after completion of the first layer. The snapshot (c) shows that the growth of the second layer is constrained by both types of boundaries. The border lines of the main rotational domains are indicated in all images.

situations are compared. In Figure 7a, various first layer domains are seen just before completion of the monolayer. The two arrows indicate two situations where growing islands merge forming domain boundaries (see also Supporting Information). In each case, the two islands form on the same substrate terrace but come from different nucleation centers moving together. From the colored image of the saturated monolayer of Figure $7 \mathrm{~b}$, one can derive that the two domains that merged at the red arrow are of the same rotational type, while those at the yellow arrow are of different rotational type. In Figure $7 c$, which shows the growth of the second layer (here the total coverage is about 1.4 ML), we find that both types of domain boundaries may act as constraints for the growth of the second layer. Hence, we conclude that at least in some cases the boundaries of translational domains also limit the size of the domains of the second layer.

3.4. Growth Kinetics. First Layer. From all observations, we can unambiguously derive that the deposited (hot) PTCDA molecules diffuse over large distances before they are attached to a fixed adsorption site. Molecules in this mobile (diffusion) state can be described as weakly bound precursor state leading to a two-dimensional "lattice gas" of PTCDA molecules that temporarily keeps the equilibrium between arriving and adsorbing molecules. The diffusion process is generally not limited by substrate steps (see refs 11 and 41 and below) or step bunches indicating that Ehrlich-Schwoebel ${ }^{42-44}$ barriers do not exist or are not sufficiently high to limit the diffusion significantly. The mobile precursor molecules diffuse until they find a nucleation center at which they can be attached and be more strongly bound to the substrate and to neighboring adsorbates. The adsorption bond on the Ag substrate is known to be chemisorptive involving significant rehybridization of the frontier orbitals ${ }^{10,45,46}$ and leading to a measurable distortion of the entire molecule. ${ }^{47,48}$ As shown above, the adsorption first occurs on steps and step bunches followed by nucleation at point defects and step bunches. The diffusion lengths are several micrometers; the distances between nucleation centers are in the range of $1 \mu \mathrm{m}$ on smooth surface areas but strongly depend on the defect density of the substrate surface ${ }^{41}$ and also on the deposition rate and temperature (not tested here).

Second Layer. For the second and higher layers, the diffusion length in the precursor state apparently increases. Although it can hardly be quantified, we can give an estimate: our movies (Supporting Information, see also ref 11) show that often nothing happens within a range of a few tens of micrometers for a while before nucleation and island growth sets in. Hence the diffusion lengths must be of the same order (several tens of micrometers). This means that step bunches or domain boundaries of the first layer do hardly play any role for the diffusion of the molecules in the second layer precursor state. This situation of course changes after nucleation: the islands of attached (bound) molecules grow on top of the first layer domains but their growth is limited by domain boundaries of the first layer and hence also by steps and step bunches of the substrate surface. The size of adsorbed microcrystallites (i.e., highly ordered epitaxial domains) and hence the morphology of the growing epitaxial layer thus strongly depends on the morphology of the substrate surface. This determines not only the density of nucleation centers ${ }^{41}$ but also the maximum size of the domains due to the size limitations induced by steps and step bunches that not only influence the first but also the second (and probably higher) layer(s).

Comparison of Both Layers. One may ask whether the island growth is similar in both layers, or whether distinct differences exist. Figure 8 compares the growth of islands in both layers, the images of the upper row represent the first,
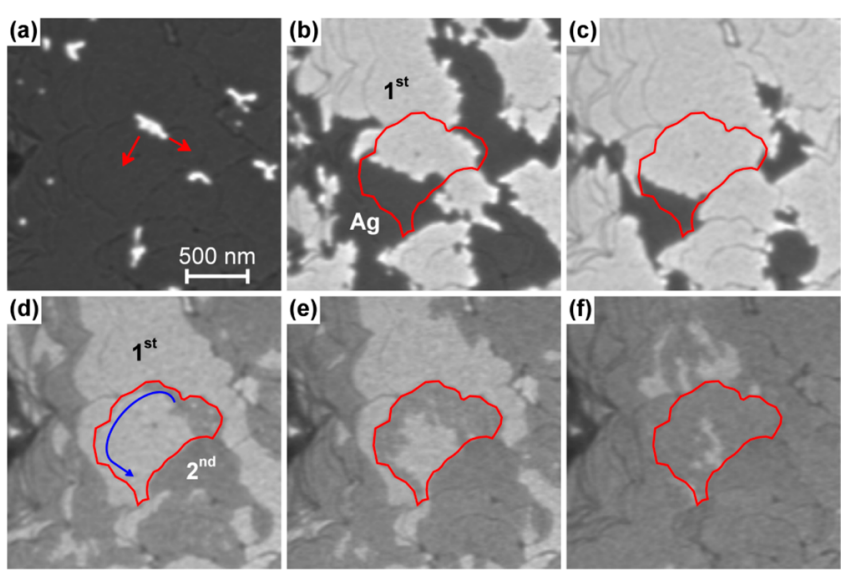

Figure 8. $(a-c)$ Growth of the first layer of PTCDA on $\operatorname{Ag}(111)$ and $(d-f)$ of the second layer on top of the first. All images display the same sample region, and the same terrace is highlighted in red in all images except (a). The growth of the first layer starts from a nucleation center (see panel a) and proceeds in all directions (indicated by red arrows) until the terrace is completely covered. In the bottom row, the second layer grows first along the steps (blue arrow in (d)) and then toward the inner part of the terrace. The coverages of the images are $0.05,0.62,0.81,1.33,1.47$, and $1.79 \mathrm{ML}$ for $(a-f)$, respectively. 
those of the lower row represent the second layer. In the first layer, islands that nucleated at point defects apparently grow in all directions equally (Figure $8 \mathrm{a}$ ). For those that start at step bunches, the situation is less clear but there appears to be a general (not unique) trend: in the first layer the island starts at one edge of a terrace (e.g., a step bunch) and uniformly grows on this terrace (an example of a terrace is indicated as thin red line in Figure 8) between its limits (step bunches) until the terrace is completely covered (Figure $8 b, c)$. In the second layer, the growing island first "wets" a large fraction of the edge of the terrace (i.e., it grows along the edge) before it fills the rest (center) of the terrace (Figure $8 \mathrm{~d}-\mathrm{f}$ ).

The observation that the diffusion length of the precursor state is much larger than the size of most terraces and domains has further consequences: the domains on the various terraces do not grow parallel but sequentially. Figure 9 compares the
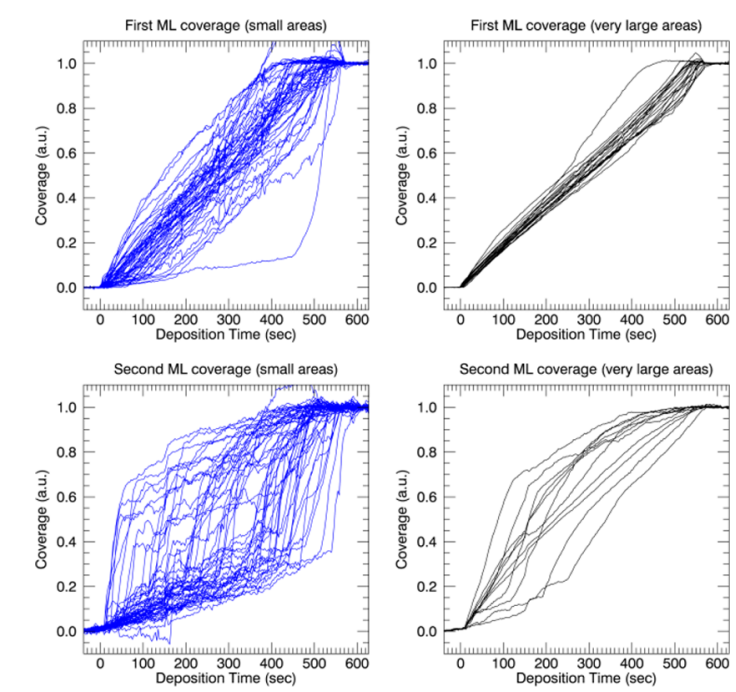

Figure 9. Development of the coverages of a number of substrate terraces as a function of deposition time for the first layer (top figures) and for the second layer (bottom figures), respectively. Small- and medium-sized terraces (left column) are distinguished from large terraces (right column). Each terrace from a region of about $15 \times 15$ $\mu \mathrm{m}^{2}$ is represented by one curve.

local coverage development of a large number of islands for the first (upper row) and the second layer (lower low) and distinguishes between small (left column) and large terraces/ islands (right column). For the first layer, we see that some of the small terraces are immediately covered by a growing island while other islands start growing with a delayed onset, and that the first islands are completed long before the others. This evaluation corroborates the above finding that the diffusion length in the precursor state is much larger than the size of the small terraces. On large terraces, however, several nucleation centers exist and hence several islands start growing simultaneously until the terrace is completely covered. Hence there is no delayed onset (and completion) of island growth. This observation is again compatible with our finding that several nucleation centers exist on large terraces and that the diffusion length in the precursor state is much larger than the distance between nucleation centers.

In the second layer, the behavior changes significantly. The spread of delayed onset (and completion) increases by a factor of 2-3 leading to the situation that some domains are already completed long before others have started to grow. On large terraces, there is now also a spread of delayed onset (completion) that is compatible with the finding that the number of nucleation centers has decreased ${ }^{41}$ and that often also on large terraces only one nucleation center exists. This behavior can be easily understood if we consider the much larger diffusion length in the precursor state and the much weaker adsorption bond of the bound molecule in the second as compared to the first layer.

\section{CONCLUDING REMARKS}

Utilizing the in situ high-resolution microscopic surface technique LEEM, we have investigated in quite some detail the initial growth behavior of the flat organic molecule PTCDA on a $\operatorname{Ag}(111)$ surface. By choosing the proper electron energy, we could optimize the contrast between the different layers and hence were able to clearly distinguish between these. Furthermore, by using dark-field contrast we were able to disentangle the six different rotational/mirror domains such that we could follow the domain growth in hitherto inaccessible detail. Thus, we could derive the influence of step and step bunches of their direction and of the terrace size on the nucleation process and on the size and on the rotational orientation of the domains. The influence of domain boundaries could be investigated in some detail.

The major results concerning the growth behavior of epitaxial organic layers are the following:

- The size of single (rotational/mirror) domains with a superstructure that is commensurate to that of the substrate surface can be in the order of several micrometers.

- The domain size strongly depends on the substrate morphology: the higher the density of step bunches the smaller are the substrate terraces and hence the smaller are the single adsorbate domains.

- Thus, the morphology of the substrate surface appears to be at least as important for the "quality" of the epitaxial layer as the preparation parameters temperature and deposition rate. Here "quality" means the size of single crystallites or, equivalently, number of defects (e.g., domain boundaries).

- The domain structure of the second layer is approximately equal to that of the first layer; domain boundaries of the latter limit the domain sizes of the former. In only a few cases, two or three domains grow in the second layer on only one domain of the first layer, thus slightly increasing the number of domains in the second layer.

- The deposited molecules diffuse over large distances in a kind of precursor state before they adsorb on a fixed adsorption site at the rim of an island. These distances are estimated to be in the range of several micrometers for the first layer and several tens of micrometers for the second layer. The large diffusion lengths are responsible for the large domain sizes.

- The initial adsorption of PTCDA islands occurs in the following way: first adsorption on steps and step bunches, followed by nucleation at point defects and step bunches, and growth of the nucleated islands.

- The average distance between nucleation centers is about 1 $\mu \mathrm{m}$ in the first layer; thus, several nucleation centers exist on large substrate terraces leading to different domains on the same terrace.

- In the second layer, the distances between the initial nucleation centers is significantly larger but the domain sizes are of the same order as in the first layer because the size of the second layer domains is determined by domain boundaries of the first layer. 
We conclude that with the knowledge from the present investigation it should be possible to optimize the growth conditions such that epitaxial layers with very large domains and few defects can be obtained. For other systems, the growth behavior has to be studied in similar detail because the bonding between molecule and substrate as well as among the molecules, the symmetry of the molecule, and the morphology of the surface play a crucial role and hence may alter the growth conditions significantly. The LEEM method including darkfield experiments is ideally suited for such studies.

\section{ASSOCIATED CONTENT}

\section{S Supporting Information}

The Supporting Information is available free of charge on the ACS Publications website at DOI: 10.1021/acs.jpcc.6b06781.

Growth of the first layer of PTCDA on the $\operatorname{Ag}(111)$ surface, observed in LEEM; the field of view is $9.6 \mu \mathrm{m}$ (AVI)

Growth of the second layer of PTCDA on the $\operatorname{Ag}(111)$ surface, observed in LEEM; the field of view is $9.6 \mu \mathrm{m}$ (AVI)

Second layer growth but here each frame is superimposed with the color-coded structure of the rotational domains in the first monolayer; the field of view is 9.6 $\mu \mathrm{m}$ (AVI)

\section{AUTHOR INFORMATION}

\section{Corresponding Author}

*E-mail: schmidtt@fhi-berlin.mpg.de. Phone: +49 308062 14671.

\section{Notes}

The authors declare no competing financial interest.

\section{ACKNOWLEDGMENTS}

The authors thank Rainer Fink, Moritz Sokolowski, and Achim Schöll for valuable discussions. They highly appreciate the technical support by the BESSY-II staff, especially Matthias Mast, Fred Senf, and Christian Jung. Financial support by the Federal German Ministry of Education and Research (BMBF) under the contract $05 \mathrm{KS} 4 \mathrm{WWB} / 4$ as well as by the MaxPlanck Society is gratefully acknowledged.

\section{REFERENCES}

(1) Brabec, C.; Lane, P.; Kafafi, Z. H. Guest Editorial: Special Section on Organic Photovoltaics. J. Photonics Energy 2011, 1, 011199.

(2) Brabec, C. J.; Heeney, M.; McCulloch, I.; Nelson, J. Influence of Blend Microstructure on Bulk Heterojunction Organic Photovoltaic Performance. Chem. Soc. Rev. 2011, 40, 1185-99.

(3) Muccini, M. A Bright Future for Organic Field-Effect Transistors. Nat. Mater. 2006, 5, 605-13.

(4) Geffroy, B.; Le Roy, P.; Prat, C. Organic Light-Emitting Diode (OLED) Technology: Materials, Devices and Display Technologies. Polym. Int. 2006, 55, 572-582.

(5) Schneider, M.; Umbach, E.; Sokolowski, M. Growth-Dependent Optical Properties of 3,4,9,10-Perylenetetracarboxylicacid-Dianhydride (PTCDA) Films on Ag(111). Chem. Phys. 2006, 325, 185-192.

(6) Eremtchenko, M.; Schaefer, J. A.; Tautz, F. S. Understanding and Tuning the Epitaxy of Large Aromatic Adsorbates by Molecular Design. Nature 2003, 425, 602-5.

(7) Kilian, L.; Umbach, E.; Sokolowski, M. Molecular Beam Epitaxy of Organic Films Investigated by High Resolution Low Energy Electron Diffraction (SPA-LEED): 3,4,9,10-Perylenetetracarboxylicacid-Dianhydride (PTCDA) on Ag(111). Surf. Sci. 2004, 573, 359-378.
(8) Glöckler, K.; Seidel, C.; Soukopp, A.; Sokolowski, M.; Umbach, E.; Bohringer, M.; Berndt, R.; Schneider, W. D. Highly Ordered Structures and Submolecular Scanning Tunnelling Microscopy Contrast of PTCDA and DM-PBDCI Monolayers on $\operatorname{Ag}(111)$ and $\mathrm{Ag}(110)$. Surf. Sci. 1998, 405, 1-20.

(9) Taborski, J.; Väterlein, P.; Dietz, H.; Zimmermann, U.; Umbach, E. NEXAFS Investigations on Ordered Adsorbate Layers of Large Aromatic Molecules. J. Electron Spectrosc. Relat. Phenom. 1995, 75, 129-147.

(10) Zou, Y.; Kilian, L.; Schöll, A.; Schmidt, T.; Fink, R.; Umbach, E. Chemical Bonding of PTCDA on Ag Surfaces and the Formation of Interface States. Surf. Sci. 2006, 600, 1240-1251.

(11) Marchetto, H.; Schmidt, T.; Groh, U.; Maier, F. C.; Lévesque, P. L.; Fink, R. H.; Freund, H. J.; Umbach, E. Direct Observation of Epitaxial Organic Film Growth: Temperature-Dependent Growth Mechanisms and Metastability. Phys. Chem. Chem. Phys. 2015, 17, 29150-60.

(12) Krause, B.; Dürr, A. C.; Ritley, K.; Schreiber, F.; Dosch, H.; Smilgies, D. Structure and Growth Morphology of an Archetypal System for Organic Epitaxy: PTCDA on Ag(111). Phys. Rev. B: Condens. Matter Mater. Phys. 2002, 66, 235404.

(13) Krause, B.; Schreiber, F.; Dosch, H.; Pimpinelli, A.; Seeck, O. H. Temperature Dependence of the 2d-3d Transition in the Growth of PTCDA on $\operatorname{Ag}(111)$ : A Real-Time X-Ray and Kinetic Monte Carlo Study. Europhys. Lett. 2004, 65, 372-378.

(14) Fink, R.; Weiss, M. R.; Umbach, E.; Preikszas, D.; Rose, H.; Spehr, R.; Hartel, P.; Engel, W.; Degenhardt, R.; Wichtendahl, R.; et al. SMART: A Planned Ultrahigh-Resolution Spectromicroscope for BESSY II. J. Electron Spectrosc. Relat. Phenom. 1997, 84, 231-250.

(15) Wichtendahl, R.; Fink, R.; Kuhlenbeck, H.; Preikszas, D.; Rose, H.; Spehr, R.; Hartel, P.; Engel, W.; Schlögl, R.; Freund, H.-J.; et al. SMART: An Aberration-Corrected XPEEM/LEEM with Energy Filter. Surf. Rev. Lett. 1998, 5, 1249-1256.

(16) Schmidt, T.; Groh, U.; Fink, R.; Umbach, E.; Schaff, O.; Engel, W.; Richter, B.; Kuhlenbeck, H.; Schlögl, R.; Freund, H.-J. XPEEM with Energy-Filtering: Advantages and First Results from the SMART Project. Surf. Rev. Lett. 2002, 9, 223-232.

(17) Rose, H.; Preikszas, D. Outline of a Versatile Corrected LEEM. Optik 1992, 92, 31-44.

(18) Rose, H.; Preikszas, D. Time-Dependent Perturbation Formalism for Calculating the Aberrations of Systems with Large Ray Gradients. Nucl. Instrum. Methods Phys. Res., Sect. A 1995, 363, 301315.

(19) Preikszas, D.; Rose, H. Correction Properties of Electron Mirrors. J. Electron Microsc. 1997, 46, 1-9.

(20) Schmidt, Th.; Marchetto, H.; Lévesque, P. L.; Groh, U.; Maier, F.; Preikszas, D.; Hartel, P.; Spehr, R.; Lilienkamp, G.; Engel, W.; et al. Double Aberration Correction in a Low-Energy Electron Microscope. Ultramicroscopy 2010, 110, 1358-61.

(21) Schmidt, T.; Sala, A.; Marchetto, H.; Umbach, E.; Freund, H. J. First Experimental Proof for Aberration Correction in XPEEM: Resolution, Transmission Enhancement, and Limitation by Space Charge Effects. Ultramicroscopy 2013, 126, 23-32.

(22) Bauer, E. Low Energy Electron Microscopy. Rep. Prog. Phys. 1994, 57, 895-938.

(23) Altman, M. S.; Chung, W. F.; Liu, C. H. LEEM Phase Contrast. Surf. Rev. Lett. 1998, 5, 1129-1141.

(24) Bauer, E. LEEM Basics. Surf. Rev. Lett. 1998, 5, 1275-1286.

(25) Kennedy, S. M.; Schofield, N. E.; Paganin, D. M.; Jesson, D. E. Wave Optical Treatment of Surface Step Contrast in Low-Energy Electron Microscopy. Surf. Rev. Lett. 2009, 16, 855-867.

(26) Pang, A. B.; Müller, T.; Altman, M. S.; Bauer, E. Fourier Optics of Image Formation in LEEM. J. Phys.: Condens. Matter 2009, 21, 314006.

(27) Schramm, S. M.; Pang, A. B.; Altman, M. S.; Tromp, R. M. A Contrast Transfer Function Approach for Image Calculations in Standard and Aberration-Corrected LEEM and PEEM. Ultramicroscopy 2012, 115, 88-108. 
(28) Chung, W. F.; Altman, M. S. Step Contrast in Low Energy Electron Microscopy. Ultramicroscopy 1998, 74, 237-246.

(29) Kilian, L.; Hauschild, A.; Temirov, R.; Soubatch, S.; Schöll, A.; Bendounan, A.; Reinert, F.; Lee, T.-L.; Tautz, F. S.; Sokolowski, M.; et al. Role of Intermolecular Interactions on the Electronic and Geometric Structure of a Large Pi-Conjugated Molecule Adsorbed on a Metal Surface. Phys. Rev. Lett. 2008, 100, 136103.

(30) Sellam, F.; Schmitz-Hübsch, T.; Toerker, M.; Mannsfeld, S.; Proehl, H.; Fritz, T.; Leo, K.; Simpson, C.; Müllen, K. LEED and STM Investigations of Organic-Organic Heterostructures Grown by Molecular Beam Epitaxy. Surf. Sci. 2001, 478, 113-121.

(31) Schmitt, S.; Schöll, A.; Umbach, E. Long-Range Surface Faceting Induced by Chemisorption of PTCDA on Stepped Ag(111) Surfaces. Surf. Sci. 2016, 643, 59-64.

(32) Ehrlich, G. The Mechanism of Chemisorption on Metals. J. Phys. Chem. Solids 1956, 1, 3.

(33) Kisliuk, P. The Sticking Probabilities of Gases Chemisorbed on the Surfaces of Solids. J. Phys. Chem. Solids 1957, 3, 95-101.

(34) Kisuuk, P. The Sticking Probabilities of Gases Chemisorbed on the Surfaces of Solids 0.2. J. Phys. Chem. Solids 1958, 5, 78-84.

(35) Taylor, J. B.; Langmuir, I. The Evaporation of Atoms, Ions and Electrons from Caesium Films on Tungsten. Phys. Rev. 1933, 44, 423458.

(36) Menzel, D. Thermal Desorption. In Chemistry and Physics of Solid Surfaces IV; Vanselow, R., Howe, R., Eds.; Springer Berlin Heidelberg: Berlin, 1982; pp 389-406.

(37) Kilian, L.; Weigand, W.; Umbach, E.; Langner, A.; Sokolowski, M.; Meyerheim, H. L.; Maltor, H.; Cowie, B. C. C.; Lee, T.; Bäuerle, P. Adsorption Site Determination of a Large Pi-Conjugated Molecule by Normal Incidence X-Ray Standing Waves: End-Capped Quaterthiophene on $\mathrm{Ag}(111)$. Phys. Rev. B: Condens. Matter Mater. Phys. 2002, 66, 075412.

(38) Telieps, W.; Mundschau, M.; Bauer, E. Dark Field Imaging with LEEM. Optik 1987, 77, 93-97.

(39) Lovinger, A. J.; Forrest, S. R.; Kaplan, M. L.; Schmidt, P. H.; Venkatesan, T. Structural and Morphological Investigation of the Development of Electrical-Conductivity in Ion-Irradiated Thin-Films of an Organic Material. J. Appl. Phys. 1984, 55, 476-482.

(40) Möbus, M.; Karl, N.; Kobayashi, T. Structure of PeryleneTetracarboxylic-Dianhydride Thin-Films on Alkali-Halide Crystal Substrates. J. Cryst. Growth 1992, 116, 495-504.

(41) Marchetto, H.; Groh, U.; Schmidt, T.; Fink, R.; Freund, H. J.; Umbach, E. Influence of Substrate Morphology on Organic Layer Growth: PTCDA on $\operatorname{Ag}(111)$. Chem. Phys. 2006, 325, 178-184.

(42) Ehrlich, G.; Hudda, F. G. Atomic View of Surface Self-Diffusion - Tungsten on Tungsten. J. Chem. Phys. 1966, 44, 1039.

(43) Schwoebel, R. L.; Shipsey, E. J. Step Motion on Crystal Surfaces. J. Appl. Phys. 1966, 37, 3682.

(44) Schwoebel, R. L. Step Motion on Crystal Surfaces 0.2. J. Appl. Phys. 1969, 40, 614.

(45) Ziroff, J.; Forster, F.; Schöll, A.; Puschnig, P.; Reinert, F. Hybridization of Organic Molecular Orbitals with Substrate States at Interfaces: PTCDA on Silver. Phys. Rev. Lett. 2010, 104, 233004.

(46) Wiessner, M.; Ziroff, J.; Forster, F.; Arita, M.; Shimada, K.; Puschnig, P.; Schöll, A.; Reinert, F. Substrate-Mediated BandDispersion of Adsorbate Molecular States. Nat. Commun. 2013, 4, 1514.

(47) Hauschild, A.; Karki, K.; Cowie, B. C.; Rohlfing, M.; Tautz, F. S.; Sokolowski, M. Molecular Distortions and Chemical Bonding of a Large Pi-Conjugated Molecule on a Metal Surface. Phys. Rev. Lett. 2005, 94, 036106.

(48) Stadtmüller, B.; Lüftner, D.; Willenbockel, M.; Reinisch, E. M.; Sueyoshi, T.; Koller, G.; Soubatch, S.; Ramsey, M. G.; Puschnig, P.; Tautz, F. S.; et al. Unexpected Interplay of Bonding Height and Energy Level Alignment at Heteromolecular Hybrid Interfaces. Nat. Commun. 2014, 5, 3685. 